\title{
Echocardiographic diagnosis and evaluation of cardiomyopathies: idiopathic hypertrophic subaortic stenosis, Chagas' heart disease and endomyocardial fibrosis
}

\author{
OtTo HeRnández-PiERETTI \\ M.D., F.A.C.A.
}

\begin{abstract}
Summary
Echocardiographic investigations on patients with hypertrophic cardiomyopathy with obstruction have been detailed and compared with the changes found in sixty patients with chronic Chagas' 'cardiomyopathy'. These changes are similar to those encountered in congestive cardiomyopathy.

Endomyocardial fibrosis is rare in Venezuela, but six patients have been found in that country and the echocardiographic changes in one of these patients has been included in this study.
\end{abstract}

ECHOCARDIOGRAPHY has developed into a most useful clinical non-invasive tool. Its application to the study of cardiomyopathies represents a major contribution to the knowledge of this clinical condition (Braunwald et al., 1964; Dinsmore, Sanders and Harthorne, 1966; Simon, Ross and Gault, 1967).

An extensive literature concerning idiopathic hypertrophic subaortic stenosis (IHSS) reveals the value of echocardiography to determine anatomical and functional alterations of this disease (Braunwald and Aygen, 1963; Braunwald, Brockenbrough and Morrow, 1962; Goodwin, 1974; Goodwin and Oakley, 1972; Henry et al., 1974; Henry, Clark and Epstein, 1973a, b; Henry et al., 1973c; Henry et al., 1975; Maron et al., 1974; Popp and Harrison, 1969; Reis et al., 1974; Shah, Gramiak and Kramer, 1969; Shah et al., 1971).

\section{Echocardiographic findings in IHSS}

These can be summarized as follows:

(1) Septal thickness is increased (N 9-11 mm) as well as ratio septal thickness/posterior wall thickness $(\mathrm{N}<1 \cdot 3)$ (Fig. 1).

(2) Abnormal systolic anterior motion (SAM) of the mitral valve echogram which is the most characteristic sign of IHSS (Shah et al., 1969). This abnormal anterior motion of mitral leaflets may in some cases contact the interventricular septum causing obstruction of the left ventricular outflow tract (Fig. 2). However, various degrees of obstruction may occur. SAM is better recorded at the free border of mitral valve leaflets and decreases when a scanning is made towards mitral annulus and subaortic region. Cases of isolated septal asymmetric hypertrophy without evidence of obstruction of left ventricular outflow tract have also been seen. However, pharmacological tests should be performed in these cases

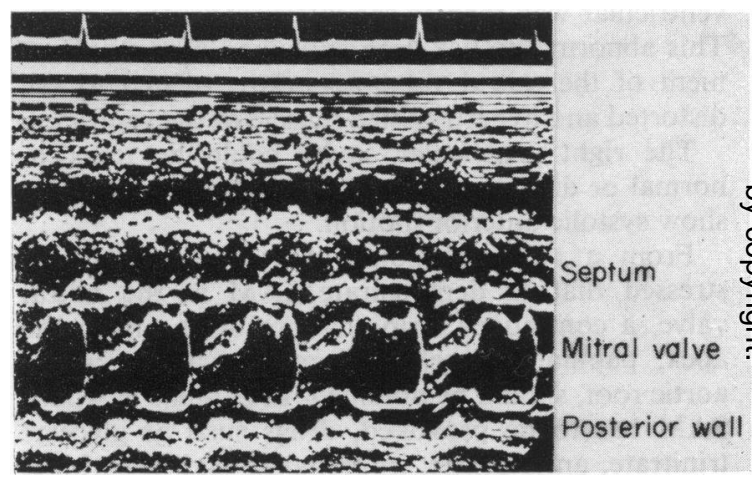

FIG. 1. Asymmetric septal hypertrophy with moderate obstruction of the left ventricular outflow tract. Septal thickness is $18 \mathrm{~mm}$. Systolic anterior motion of mitral valve is observed.

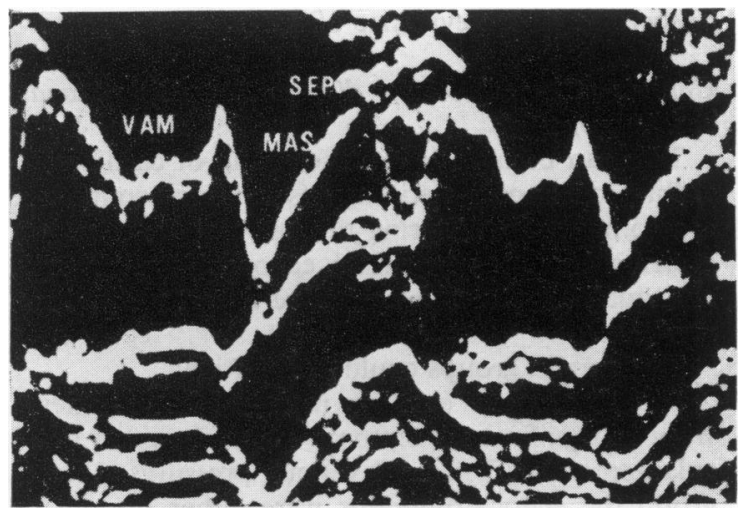

Fig. 2. Typical systolic anterior motion of mitral valve echogram (SAM = MAS). Anterior leaflet mitral valve echo reaches interventricular septum (IHSS). 
using drugs which are capable of increasing the SAM. This spectrum has been studied by Henry et al. (1973b), who have classified this condition as follows: (i) septal hypertrophy without obstruction. (ii) Septal hypertrophy with provoked obstruction. (iii) Obstructive forms of asymmetric septal hypertrophy.

(3) Aortic echograms also show alterations in IHSS. The aortic valve opens normally in systole; afterwards, the opening position is interrupted as the aortic cusps assume a near-closed position in early systole, and re-open later in systole. This pattern represents the aortic blood flow transient reduction which occurs in early or mid-systole as a result of mechanical subaortic dynamic obstruction.

(4) Another echocardiographic feature which has been reported in IHSS is an abnormal anterior displacement of the closure point of the mitral valve. ('C' point of mitral echogram) (Henry et al., 1973b). Normally this point is closer to the posterior left ventricular wall than to the interventricular septum. This abnormality has been explained by a displacement of the mitral valve apparatus related to the distorted and hypertrophied muscle of the ventricles.

The right ventricular cavity could be reduced, normal or dilated, and the tricuspid valve may also show systolic anterior motion.

From a technical point of view it should be stressed that to demonstrate SAM at the mitral valve, a continuous strip chart recording from the apex, papillary muscle and mitral valve towards aortic root, should be obtained. In patients in whom SAM has to be provoked, drugs such as glyceryl trinitrate, amyl nitrite, isoprenaline and isosorbide, should be used. The Valsalva manoeuvre and observation of the systolic portion of the mitral valve echo in post-extrasystolic beats may reveal the SAM, but it should be stressed that subaortic stenosis with a characteristic SAM of the mitral valve has been observed without evidence of septal hypertrophy.

\section{Chronic Chagas' heart disease (chronic Chagas' cardiomyopathy)}

This can be included in the group of cardiomyopathies of known aetiology (secondary congestive cardiomyopathy). During the last three years, sixty patients have been studied echocardiographically, and qualitative and quantitative aspects have been analysed.

Three main groups could be identified.

The first group consists mainly of out-patients without significant symptoms, no evidence of heart enlargement and normal venous pressures. In this group the echocardiograms were either normal or with alterations of the mitral valve motion pattern related to extrasystoles, and localized areas in the septum with poor motion. Septal and left ventricular posterior walls were of normal width. In two patients of this group posterior wall excursion was diminished.

The second group included patients with moderate heart failure, frequent extrasystoles and moderate cardiomegaly. In this group, the right and/or left ventricle, and the left ventricular outflow tract, were seen to be dilated. Mitral valve echoes were reduced in amplitude with easy and frequent recording of both cusps (Fig. 3). The posterior mitral valve leaflet echoes are usually easy to obtain, showing its characteristic ' $W$ ' motion pattern, and well defined echoes quite distant from the posterior left ventricular wall, indicating a dilated left ventricular chamber. Septa showed abnormal motion pattern in most cases (hypokinesis, akinesis or paradoxical motion). Left ventricular wall also showed hypokinesis, occasionally. Its thickness is either normal or e diminished and, in a few cases, an indication of hypertrophy.

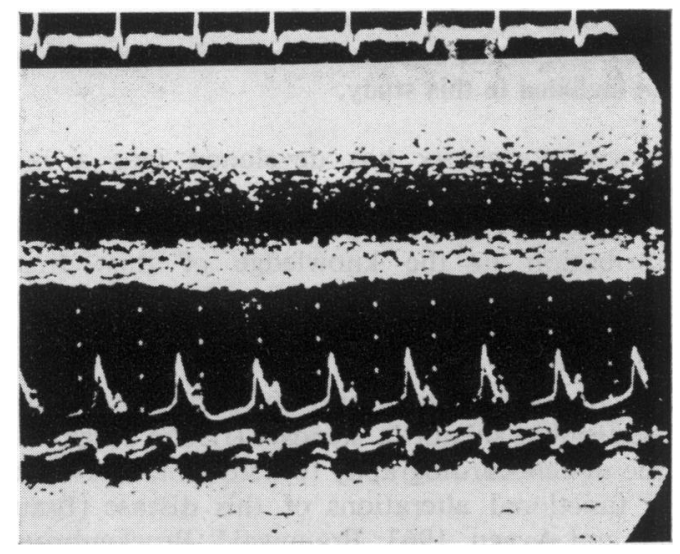

FIG. 3. Chronic Chagas' heart disease in heart failure (Group II). Right and left ventricles are dilated. Frequent and easy recording of both mitral cusps, 'flat' (akinetic) interventricular septum, hypokinetic left ventricular wall and dilated left ventricular outflow tract are observed.

The third group included patients with severe congestive heart failure and cardiomegaly. The interventricular septum is usually remarkably thin and shows no motion ('flat' septum) or paradoxical movement. Left ventricular posterior walls also show poor movement and in several cases it was not possible to obtain a reliable posterior wall endocardial echo. The most striking findings at this stage are the extremely dilated right and/or left ventricular $O$ cavities with very thin walls and interventricular septa. The left ventricular outflow tracts are wide. The mitral valve shows a low cardiac output motion pattern with diminished excursion and well defined 


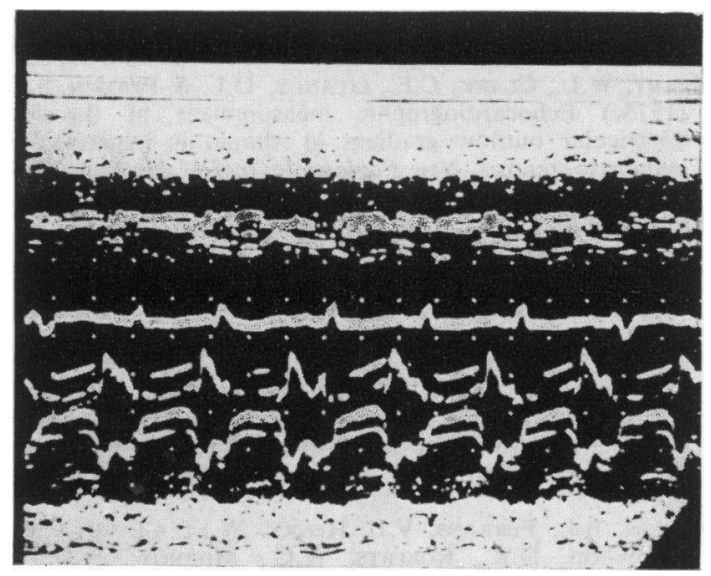

FIG. 4. Chronic Chagas' heart disease in refractory sèvere heart failure (Group III). Left ventricular cavity shows marked dilatation. Interventricular septum is very thin and akinetic. Left ventricular posterior wall shows poor motion. Easy recording of both mitral cusps with multiple echoes in systole. Left ventricular outflow tract is also dilated.

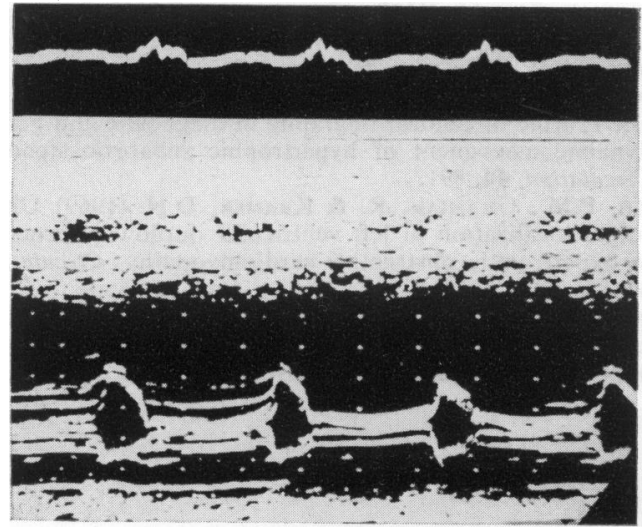

Fig. 5. Low cardiac output mitral echo pattern with poor excursion and multiple systolic echoes with flat or horizontal C-D. Left ventricle is dilated. Both interventricular septum and left ventricular posterior wall are thin and akinetic (Chagas' heart disease).

echoes. Both cusps can easily be recorded (Fig. 4). The systolic portion of the mitral echogram is horizontal and frequently shows multiple linear echoes arising in chordae or papillary muscles (Fig. 5). The A-C slope is slow with recording of a notch or bulge on its descent in a few cases. One characteristic sign is the abnormal early diastolic pattern of the mitral valve echo, which may be observed in severely congested patients. Early diastolic slopes may move rather rapidly; however, they may be either interrupted or followed by an additional diastolic bulge indicating a reopening motion of the mitral valve. A number of echocardiograms of patients with Chagas' heart disease were reviewed with $\mathrm{Dr} \mathbf{H}$. Feigenbaum, who stressed the diagnostic value of this abnormal diastolic bulge and extremely thin ventricular walls as a characteristic sign of severe Chagas' cardiomyopathy. This abnormal bulge has not been observed in other types of congestive cardiomyopathies, and could be related to an abnormal early diastolic filling pattern of the left ventricle.

In other severe cases, echoes arising in the aortic cusps are easy to record, showing continuous well defined echoes both in diastole and systole, which converge in mid- and late systole. The aortic walls show diminished systolic motion.

From the quantitative point of view, the author measured right ventricular internal diameter, left ventricular internal diameter (in late diastole and late systole), septal and posterior wall thickness, opening amplitude of the mitral valve, excursion amplitude of septum and posterior wall, normalized mean velocity of circumferential shortening of the left ventricle, ventricular volume, ejection fraction, left ventricular outflow tract diameter, aortic root diameter, aortic cusp opening amplitude, E-F and A-C slopes of mitral valve echoes, left atrium and mean velocity of left ventricular posterior wall. Measurements of ventricular function revealed a poor left ventricular function with an increased end diastolic pressure.

Moderate pericardial effusion was observed in a few cases.

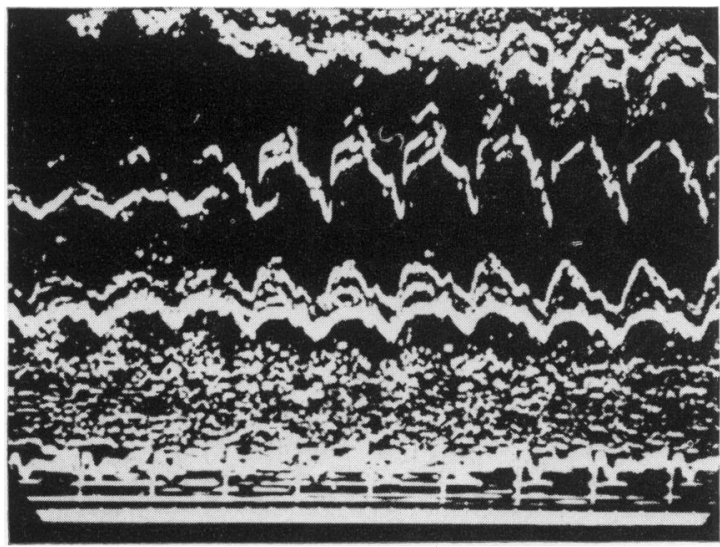

FIG. 6. Echocardiogram of a patient with endomyocardial fibrosis proved by post-mortem study. A strong and continuous echo is observed on right septal endocardium. Right ventricular cavity is diminished. Despite an increase in damping control, the strong echo arising in right septal endocardium remains. 


\section{Endomyocardial fibrosis}

This is an uncommon disease in Venezuela. However, six cases with post-mortem study have been reported. The author had the opportunity to study the echocardiogram recorded from a patient who had been studied clinically by J. J. Puigbó and associates. The echocardiogram showed an abnormal strong echo arising from the right ventricular septal endocardium. Increasing damping control diminished or made left ventricular septal echoes disappear. However, the strong echo from the right ventricular septum remained (Fig. 6). Post-mortem study confirmed the diagnosis of endomyocardial fibrosis.

\section{References}

Braunwald, E. \& Aygen, M.M. (1963) Idiopathic myocardial hypertrophy without congestive heart failure or obstruction to blood flow. American Journal of Medicine, 35, 7.

Braunwald, E., Brockenbrough, E.C. \& Morrow, A.G. (1962) Hypertrophic subaortic stenosis-a broadened concept. Circulation, 26, 161.

Baunwald, E., Lambrew, C.T., Rockoff, S.D., Ross, J., JR \& Morrow, A.G. (1964) Idiopathic hypertrophic subaortic stenosis. I. A description of the disease based upon an analysis of 64 patients. Circulation, 30 (Suppl. 4), 3.

Dinsmore, R.E., SANDers, C.A. \& HARThORNe, J.W. (1966) Mitral regurgitation in idiopathic hypertrophic subaortic stenosis. New England Journal of Medicine, 275, 1225.

GoodwiN, J.F. (1974) Prospects and predications for the cardiomyopathies. Circulation, 50, 210.

Goodwin, J.F. \& OAKLEY, C.M. (1972) The cardiomyopathies. British Heart Journal, 34, 545.

Henry, W.L., Clark, C.E. \& Epstein, S.E. (1973a) Asymmetric septal hypertrophy: echocardiographic identification of the pathognomonic anatomic abnormality of IHSS. Circulation, 47, 225.

Henry, W.L., Clark, C.E. \& Epstein, S.E. (1973b) Asymmetric septal hypertrophy (ASH): the unifying link in the
IHSS disease spectrum: observations regarding its patho- $\bar{Z}$ genesis, pathophysiology, and course. Circulation, 47, 827. Henry, W.L., Clark, C.E., Glancy, D.L. \& Epstein, S.E. C (1973c) Echocardiographic measurement of the left ventricular outflow gradient in idiopathic hypertrophic subaortic stenosis. New England Journal of Medicine, 288, 989.

Henry, W.L., Clark, C.E., Griffith, J.M. \& Epstein, S.E. (1975) Mechanism of the left ventricular outflow obstruc- $\bar{S}$ tion in patients with obstructive asymmetric septal hyper- $\vec{\nabla}$ trophy (idiopathic hypertrophic subaortic stenosis). @ American Journal of Cardiology, 35, 337.

Henry, W.L., Clark, C.E., Roberts, W.C., Morrow, A.G. \& EPSTEIN, S.E. (1974) Differences in distribution of myo- $\vec{O}$ cardial abnormalities in patients with obstructive and non- $\overrightarrow{\vec{H}}$ obstructive asymmetric septal hypertrophy (ASH): Echo- $\vec{\omega}$ cardiographic and gross anatomic findings. Circulation, 50, 447.

Maron, B.J., Ferrans, V.J., Henry, W.L., Clark, C.E., ReDWOOD, D.R., Roberts, W.C., MorRow, A.G. \& i EPSTEIN, S.E. (1974) Differences in distribution of myo- $\omega$ cardial abnormalities in patients with obstructive and non- $\sigma$ obstructive asymmetric septal hypertrophy (ASH): Light and electron microscopic findings. Circulation, 50, 436.

POPP, R.L. \& HARRISON, D.C. (1969) Ultrasound in the diagnosis and evaluation of therapy of idiopathic hypertrophic subaortic stenosis. Circulation, 40, 905.

Reis, R.L., Bolton, M.R., King, J.F., Pugh, D.M., Dunn, M.I. \& MASON, D.T. (1974) Anterior-superior displace- $\omega$ ment of papillary muscles producing obstruction and mitral $\mathbb{D}$ regurgitation in IHSS: Operative relief by posterior- $\frac{\vec{D}}{\vec{D}}$ superior realignment of APM following ventricular septgl 3 myectomy. Circulation, 50 (Suppl. 2), 181.

Shah, P.M., Gramiak, R., Adelman, A.G. \& Wigle, E. 8 . (1971) Role of echocardiography in diagnostic and hemo $\overrightarrow{0}$ dynamic assessment of hypertrophic subaortic stenosi Circulation, 44, 891.

ShaH, P.M., GramiaK, R. \& Kramer, D.H. (1969) Ultră sound localization of left ventricular outflow obstruction in hypertrophic obstructive cardiomyopathy. Circulation, 40,3 .

Simon, A.L., Ross, J., JR \& GAULT, J.H. (1967) Angiographic anatomy of the left ventricle and mitral valve in idiopathic $\mathbb{D}$ hypertrophic subaortic stenosis. Circulation, 36, 852. 